\title{
Daily mindfulness training reduces negative impact of COVID-19 news exposure on affective well-being
}

\author{
Julia W. Y. Kam ${ }^{1}$ (1) . Javeria Javed ${ }^{1}$. Chelsie M. Hart ${ }^{1}$. Jessica R. Andrews-Hanna ${ }^{2}$ - Lianne M. Tomfohr-Madsen ${ }^{1}$. \\ Caitlin Mills ${ }^{3}$
}

Received: 6 October 2020 / Accepted: 13 June 2021 / Published online: 24 June 2021

(c) The Author(s), under exclusive licence to Springer-Verlag GmbH Germany, part of Springer Nature 2021

\begin{abstract}
COVID-19 has led to mental health adversities worldwide. The current study examined whether daily practice of brief mindfulness training has a beneficial impact on affective well-being, and mitigates the negative impact of exposure to COVID-19 news during the pandemic. Participants were randomly assigned into a mindfulness training (MT) group or a waitlist control (WC) group. Participants in the MT group practiced guided mindfulness meditation for a minimum of 10 min each day for 10 days. Both groups completed questionnaires assessing well-being at baseline and after the 10-day period. We also included four ecological momentary assessments (EMA) interspersed throughout the day to measure fine-grained affective states and recent exposure to COVID-19-related news, which has been linked to negative affect. We observed an increase in positive affect in the MT group compared to the WC group in the post-training assessment. However, no group differences emerged in the other three post-training affective measures of negative affect, anxiety and depression. EMA revealed that the MT group also showed more positive affective valence than the WC group across the 10 days. Notably, the WC group reported more negative affective valence following COVID-19 news exposure, whereas the MT group was not impacted. Taken together, our study indicates brief sessions of guided mindfulness meditation during COVID-19 may boost positive affect and serve as a protective buffer against the negative impact of exposure to COVID-19-related news on affective wellbeing. These findings highlight the utility of mindfulness meditation as an accessible and cost-effective technique to elevate positive affect amidst the COVID-19 pandemic.
\end{abstract}

\section{Introduction}

Designated as a pandemic by the World Health Organization in March 2020, the coronavirus (COVID-19) has led to devastating health outcomes worldwide. Its impact on psychosocial well-being is equally far reaching; an increasing number of studies around the world have documented symptoms of depression and anxiety as a result of the pandemic in the general population (González-sanguino et al., 2020; Luo et al., 2020), and especially among health care workers (Lai et al., 2020; Romero et al., 2020). Several systematic reviews

Julia W. Y. Kam

julia.kam@ucalgary.ca

1 Department of Psychology, University of Calgary, 2500 University Drive NW, Calgary, AB, Canada

2 Department of Psychology; Cognitive Science, University of Arizona, 1503 E University Blvd, Tucson, AZ, USA

3 Department of Psychology, University of New Hampshire, 105 Main St, Durham, NH, USA and meta-analyses have substantiated these negative psychosocial impacts of COVID-19 (Luo et al., 2020; Pappa et al., 2020). Arising from uncertainty about the future, isolation due to quarantines, and health concerns among others, these adverse mental health effects may evolve into long-lasting consequences on quality of life well beyond the pandemic (Luo et al., 2020; Torales et al., 2020). Mental health professionals and scientists alike have urged for the development of timely, distance-based mental health intervention to address unmet mental health needs during COVID-19 (Holmes et al., 2020; Xiang et al., 2020).

A potential effective coping strategy for addressing mental health adversities during the pandemic may be mindfulness meditation. Although numerous characterizations exist, we refer mindfulness to the ability to focus on the present moment with openness (Brown \& Ryan, 2003) and a non-judgmental stance (Bishop et al., 2004; Kabat-Zinn, 1990). Training in mindfulness mediation has been shown to enhance attention (Basso et al., 2019; Jha et al., 2007; Tang et al., 2007) and acceptance (Cheung \& Ng, 2019), 
suggesting mindfulness is an ability that can be improved with practice. Intervention studies collectively demonstrate that practicing mindfulness meditation is beneficial for affective well-being, with systematic reviews and meta-analyses reporting moderate effect sizes on alleviating symptoms of depression and anxiety (Goyal et al., 2014; Hofmann et al., 2010; McClintock et al., 2019). When incorporated into clinical programs such as Mindfulness-Based Cognitive Therapy or Mindfulness-Based Stress Reduction, this robust effect is observed in psychiatric and medical populations (Goyal et al., 2014; Grossman et al., 2004). Practicing mindfulness meditation also enhances positive affect, well-being, and quality of life in the general population (Eberth \& Sedlmeier, 2012; Garland et al., 2015; Khoury et al., 2015). Even shortterm mindfulness practices (with training lasting no more than two weeks) delivered via smartphone apps have been shown to reduce negative affect, stress, and depressive symptoms as well as enhance positive affect (Economides et al., 2018; Flett et al., 2019; Lindsay et al., 2018; Schumer et al., 2018). These converging lines of evidence underscore the benefits of mindfulness meditation on affective well-being.

Traditionally, intervention studies have examined group differences in aggregate levels of affective well-being at the conclusion of the mindfulness training. This approach inherently assumes that affect is stable and overlooks the fluctuations in affect over time within individuals. Notably, affective experiences are hardly static; instead, they dynamically change throughout the day as a function of our personal and interpersonal experiences (Kuppens et al., 2010; Lapate \& Heller, 2020). To capture the dynamics of affect, more recent intervention studies have begun to use ecological momentary assessments (EMA) to measure the moment-to-moment changes in affect (Chin et al., 2019; Geschwind et al., 2011; E. K. Lindsay et al., 2018; Rowland et al., 2018). The advantages of this methodological approach are multifold (Shiffman et al., 2008): combined with the appropriate statistical analyses, it avoids washing out intra-individual differences across time; it enables the exploration of dynamic relationships between affect and ongoing experiences; it is ecologically valid as assessments take place in the real world. These intervention studies with EMA found that mindfulness training enhances momentary positive affect (Geschwind et al., 2011; Lindsay et al., 2018), as well as reduces momentary stress (Chin et al., 2019), and anxiety (Lahtinen \& Salmivalli, 2020). Such findings highlight the value of EMA in revealing the momentary relationship between affect and ongoing experience. As the current study aimed to assess the impact of mindfulness training on affective experiences, EMA enabled us to measure intraindividual differences in affective dynamics across time in the real world, highlighting the value of this methodological approach in mindfulness intervention research.
Of relevance to the current pandemic, an EMA approach allowed us to not only assess the dynamics of affect, but also determine the impact of exposure to COVID-19-related news on affective well-being. Negative news exposure has been linked to negative affective states (de Hoog \& Verboon, 2020; Unz et al., 2008). Evidence also points towards an additive effect in prolonged news exposure: for example, one study found that the amount of news exposure to the Boston Marathon bombings positively correlated with acute stress symptoms (Holman et al., 2014). Given the extent of daily news coverage on COVID-19 in the months following its designation as a pandemic, these findings suggest that exposure to COVID-19 news may contribute to the reported negative affect. In particular, one study found that obtaining COVID-19 information was associated with the worst emotional experience compared to other measured activities (Lades et al., 2020). Thus far, mindfulness has been shown to be beneficial for our affective well-being. This raises the question of whether mindfulness training can attenuate the negative affect associated with exposure to negative news. The implementation of an ecologically valid and brief mindfulness intervention plus a focus on COVID-19 news exposure sets our study apart from past research on mindfulness intervention.

The present study examined whether daily practice of brief mindfulness training had a positive impact on wellbeing, especially following exposure to COVID-19-related news. To address this, participants were randomly assigned into a mindfulness training (MT) group or waitlist control (WC) group. Participants in the MT group, but not the WC group, were instructed to practice mindfulness meditation for at least $10 \mathrm{~min}$ each day for 10 days. To assess the overall impact of mindfulness training on well-being, all participants completed the same set of questionnaires before and after the 10-day period. To assess momentary changes in affect during the 10 days, both groups completed four EMA surveys each day for all 10 days, reporting their momentary affect and exposure to COVID-19-related news. We assessed the impact of mindfulness meditation on changes in well-being post-training (via questionnaire responses) and throughout the study (via ecological momentary assessments), and examined these changes as a function of their exposure to COVID-19-related news.

\section{Methods}

\section{Participants}

A total of 64 participants were recruited for this study on a rolling basis. Recruitment was conducted via emails to graduate programs in several universities within North America, with our sample consisting primarily of graduate students 
and their affiliates. Data collection occurred between May 6th and May 31st, 2020. Subject recruitment ended as new COVID-19 cases began to plateau in Alberta, Canada, as that was the primary site of recruitment; other areas of recruitment (e.g. British Columbia, Canada; Ontario, Canada; New Hampshire, USA) also showed a decrease or plateau in new COVID-19 cases at the end of the recruitment period. We are not aware of any major COVID-19-related policy changes or unexpected events (beyond the recurring daily reports of COVID-19 cases) during this data collection period in the main sites of recruitment. Importantly, this sample size is comparable to a recent study that used the same delivery platform of mindfulness training (i.e. the smartphone app Headspace) for a similar duration and number of sessions (i.e. $10 \mathrm{~min}$ per session for 10 sessions), who reported medium effect sizes on its outcome measures of stress, affect and irritability (Economides et al., 2018). ${ }^{1}$ Two participants did not complete the study, resulting in a final sample of 62 participants (52 females and 10 males; age range $=19-63$ years, $M=29.9$ years, S.D. $=8.8$ ). Participant inclusion criteria included age 17-65, ability to speak and understand English, previous practice of mindfulness or meditation could not have exceeded once or more per week in the past 3 months. All participants provided informed consent and were paid for their participation in the form of e-gift certificates. The study was approved by the Conjoint Faculties Research Ethics Board at the University of Calgary.

\section{Design and procedure}

This 12-day study consisted of baseline and post-training assessments that took place one day before and after the 10-day mindfulness training period. Only one of two groups of participants completed the mindfulness training. However, both groups completed ecological momentary assessments throughout the 10-day period. We describe each component of the study in detail below.

\section{Mindfulness training}

Participants were randomly assigned to the Mindfulness Training (MT) group $(n=32)$ or the Waitlist Control (WC) group $(n=30)$. The MT group practiced guided mindfulness

\footnotetext{
${ }^{1}$ A post hoc power analysis indicated the number of subjects per group needed to achieve the effect size (Cohen's $d=0.47$ ) on the affective measure in Economides et al. (2018) is $n=13$ $(=.05,=80 \%)$. This study also included other well-being measures, such as stress and irritability (Cohen's $d=0.26-0.45$ ). If we considered all their outcomes measures in our power analysis by computing the average effect size across these measures, the number of subjects per group needed to achieve this effect size is $17(=.05,=80 \%)$.
}

training every day for 10 days. They were provided with a one-month subscription to Headspace (Santa Monica, California, USA), a smartphone application that offers various courses on guided training and has been used in previous mindfulness training studies (Bostock et al., 2019; Economides et al., 2018; Flett et al., 2019). Participants chose one of four 10-day courses, each targeting different aspects of well-being: kindness, managing anxiety, letting go of stress, or concentration. These courses all involve a core mindfulness component that focuses on cultivating attention and awareness in a nonjudgmental manner (Van Dam, 2017). Participants receive the option to practice meditating for 10 , 15 , or 20 min each day. During each session, listeners were instructed to sit comfortably and were guided through mindfulness meditation to develop a sense of mindful awareness. At the end of each session, they completed a daily postpractice survey that asked them to report on the following: 1) the duration of their training, 2) how focused they were during the training $(1=$ extremely unfocused, $4=$ extremely focused), and 3) how positive or negative they felt during the training $(1=$ extremely negative, $5=$ extremely positive). Participants received the daily post-practice survey in the morning and were asked to complete the survey upon finishing their daily session of mindfulness practice. This data is missing from one subject due to technical errors. The WC group did not participate in the mindfulness training during this period, but was offered a 1-month subscription to Headspace after the study concluded.

Baseline and post-training assessment. At the baseline assessment on Day 1 and post-training assessment on Day 12 , all participants completed the same set of online questionnaires that broadly assessed their affective well-being. Additional measures were collected on thought patterns but will not be reported here. Of relevance to this study, participants completed the Positive Affect and Negative Affect Scale (PANAS; state version), the Patient-Reported Outcomes Measurement Information System (PROMIS)-depression scale, and the PROMIS-anxiety scale. The PANAS is a 20-item scale assessing positive and negative affective states (Watson et al., 1988), where participants rate the extent to which they are currently experiencing 10 different positive and 10 different negative affective states (e.g., distressed, enthusiastic) on a 5-point Likert scale. The positive and negative affect subscales were computed as the sum of the scores across the 10 positive and negative items respectively, with possible scores on each subscale ranging from 10 to 50. Both the PROMIS depression and anxiety scales short forms consist of 8 items, and are commonly used to assess depression and anxiety in healthy and clinical populations (Cella et al., 2010; Pilkonis et al., 2011, 2014). Participants rated how often they felt this way in the last $24 \mathrm{~h}$ for each item on a 5-point Likert scale. The PROMIS depression and anxiety scales were computed as the sum of the scores 
across the 8 items in each scale, with possible scores on each scale ranging from 8 to 40 . To assess dispositional mindfulness, participants also completed the Mindful Attention and Awareness Scale (Brown \& Ryan, 2003). For each of the 15 items, participants rated on a 6-point Likert scale questions assessing their disposition to attend to present moment experiences. Finally, participants were also asked to report the frequency of COVID-19 news consumption over the 10 days at both time points as more than twice a day, one or twice a day, 2-3 times a week, or once a week.

\section{Ecological momentary assessment}

Ecological momentary assessments (EMA) consisted of four experience sampling surveys sent each day during the 10-day period. Both groups received the experience sampling surveys at four random times within a 10-h time window of their choice (inter-survey interval: mean $=144 \mathrm{~min}$, range $=131-156 \mathrm{~min}$ ). Participants were asked to choose their preferred 10-h time window during their waking hours that would best capture their natural circadian rhythm. Given the study was implemented during the early phase of the pandemic when individuals were still gradually adjusting to work from home policies, this individualized as opposed to a pre-determined 10-h time window was assumed to better capture the awake hours of each individual participant. The start time of the individualized 10-h time window ranged between 7 am and 1 pm.

These probes were sent by email with a link to an online survey via Qualtrics. Participants were asked to complete the survey within $15 \mathrm{~min}$ of receiving the email notification. Prior to the start of the study, participants were reminded to turn on their email notification on their phone so they could respond to probes within the 15-min time window. The online survey included a set of questions that asked participants to report on their current thoughts, affect, and behavior at the moment. These questions included 1) what they were currently doing, 2) how they felt at that moment (i.e., a measure of the valence of state affect rated on a 5-point Likert scale from $1=$ extremely negative to $5=$ extremely positive), 3) whether they watched or heard COVID 19-related news in the last two hours. Additional questions were included, but are not reported here as they are outside the scope of the current research questions.

To ensure that participants understood the meaning of the questions, they completed a training session after baseline assessments the day before the start of EMA. We provided definitions and example scenarios of each question. Following this, participants were given a new example scenario that they may encounter during the study and asked to answer the online survey based on the scenario. We implemented these extensive explanations and practice of completing the survey to familiarize the participants with the questions and to ensure their full understanding of them. Participants in both groups received a check-in email half-way through the study to encourage their continued participation in the study.

The following exclusion criteria were applied to the 2185 responses obtained from the 62 participants. EMA responses that took more than five minutes to complete from the start time of the survey were excluded; this led to the exclusion of $47(2.2 \%)$ responses. Responses to experience sampling probes that began more than 30 min after the probe was sent were also excluded; this led to the exclusion of $481(22.0 \%)$ responses. Finally, subjects who responded to less than $20 \%$ of their probes were excluded (Mills et al., 2018); this led to the exclusion of four subjects $(6.5 \%)$. Together, these exclusion criteria led to a final sample of 1638 responses from 58 participants.

\section{Statistical analyses}

Control analyses. To ensure that the two groups did not differ in demographics, we ran independent $t$ tests for continuous variables (i.e., age) and chi-square tests for categorical variables (i.e., gender, ethnicity and education level). We also compared past meditation experience and dispositional mindfulness between two groups using independent t-tests. Finally, we compared the two groups in their frequency of COVID-19 news consumption at baseline. To ensure training did not lead to a change in frequency of COVID-19 news consumption, we also compared the two groups at post-training assessment.

Baseline and post-training assessments. To examine group differences in outcome measures at post-training assessment on Day 12, we implemented a separate linear regression model for each of the four outcome measures: positive affect was assessed by the positive subscale of PANAS, negative affect was assessed by the negative subscale of PANAS, depression was assessed by the PROMISdepression scale and anxiety was assessed by the PROMISanxiety scale. Each linear regression model consisted of group (coded as $1=\mathrm{WC}$ and $2=\mathrm{MT}$ and specified as a factor) as the primary independent variable, age and gender as covariates, and the same outcome measure at baseline assessment to account for baseline differences. To determine whether the duration, focus and valence of daily practice of mindfulness meditation were associated with these outcome measures in the MT group, we also implemented separate linear regression models that consisted of the three mindfulness training-related variables and the same outcome measure at baseline assessment as independent variables. Each of these training-related variables was averaged across the 10 days of training within individuals prior to its inclusion in the regression models. These analyses within the MT group elucidate the dose-dependent relationship between mindfulness training experience and outcome measures. Importantly, 
such relationships would imply that any observed benefits of mindfulness meditation could not simply be accounted for by assignment to the MT group, instead any dose-dependent relationship would suggest that the experience of practicing mindfulness tracks the magnitude of benefit gained as a result of the mindfulness training.

\section{Ecological momentary assessments}

To assess group differences in momentary affect dynamics during the 10-day period, we implemented linear mixed effects regression analyses. Momentary ratings of the valence of state affect (as rated on a 5-point Likert scale) were regressed on three key fixed effects as our primary independent variables: group (coded as $1=\mathrm{WC}$ and $2=\mathrm{MT}$ and specified as a factor), exposure to COVID-19-related news (coded as $1=$ yes and $2=$ no and specified as a factor), and the group $x$ news interaction term. We included day-instudy as another fixed effect to account for changes across time. Participant was included as a random effect to account for baseline variability in state affect across participants. Inclusion of news and day-in-study as random slopes did not improve model fit, therefore only random intercepts of participants were considered in the model. The fixed effects of interest were tested using a two-tailed likelihood ratio test (i.e. Chi-square test) that compares the full models with the fixed effect of interest against the model without it. We also included age and gender as fixed effects covariates to ensure our results cannot be accounted for by these demographic variables. To determine whether the course, duration, focus and affective valence of daily practice of mindfulness meditation impacted the state affect in the MT group, we also implemented a linear mixed effects regression that consisted of the four mindfulness training-related variables as independent variables. Similar to the post-training assessments, these analyses within the MT group helps elucidate the dosedependent relationship between mindfulness training experience and state affect.

All statistical analyses were performed using R (R.1.2.5) and its subsidiary packages (car, colorspace, dplyr, ggplot2, lme4, ImerTest, parameters, plyr, sjPlot); the package parameters were used for standardizing coefficients, and the package car was used for significance testing (Fox et al., 2020).

\section{Results}

Table 1 reports the demographic information of our participants. The MT and WC groups did not differ in age, gender, ethnicity, years of education, past experience with meditation, and dispositional mindfulness $(p>0.271)$. There were also no group differences in exposure to COVID-related news at baseline and post-training assessment (baseline: $p=0.219$; post-training: $p=0.955)$. Specifically, both groups reported news consumption around once or twice a day at baseline and both groups reported consumption frequency between daily and 2-3 times a week at post-training assessment. The two groups also did not show differences in any of the outcome measures at baseline assessment: positive affect, negative affect, depression, anxiety $(p>0.166)$. The majority of participants in our study reported at least average levels of affective well-being. Only a small percentage reported two standard deviations above population mean levels of depression (8\%) and anxiety (5\%) at baseline. The two groups did not differ in the proportion of individuals who experienced clinical levels of depression and anxiety $(p>0.779)$. Statistical values and regression coefficients for each test are reported in the Supplementary Information.

\section{Post-training assessment}

To determine the overall impact of mindfulness meditation on affective well-being, we compared each of the outcome measures obtained at post-training assessment between the MT and WC groups, with baseline assessments included as covariates in the linear regression model. The MT group reported higher levels of positive affect $(p=0.039$, Cohen's $d=0.68$ ) compared to the WC group. The two groups did not differ in negative affect $(p=0.905)$, depression $(p=0.739)$, nor anxiety $(p=0.575)$. Removal of age and gender as covariates in these models did not change the direction or significance of the effects. Table 2 reports all statistical values associated with these linear regression models.

Next, we examined whether daily experiences of practicing meditation measured using the daily post-practice survey track with affective measures assessed at end-of-study within the MT group. In particular, we assessed the relationship between the type of course, duration of the practice, focus and valence during the mindfulness training reported daily immediately following practice and the affective outcome measures at end-of-study assessment. Participants who reported higher levels of positive affect following each mindfulness session also reported higher levels of positive affect at post-training assessment $(p=0.025)$. None of the training-related variables predicted variance in negative affect, depression, or anxiety. Details of these analyses are reported in Supplementary Information.

\section{Ecological momentary assessments}

On average, the MT group responded to $28.12(70.3 \%)$ probes, and the WC group responded to 28.34 (70.9\%) probes that fulfilled our inclusion criteria. To ascertain the day-to-day impact of mindfulness training on affective dynamics, we examined group differences in state affect obtained from EMA. Analyses revealed significantly more 


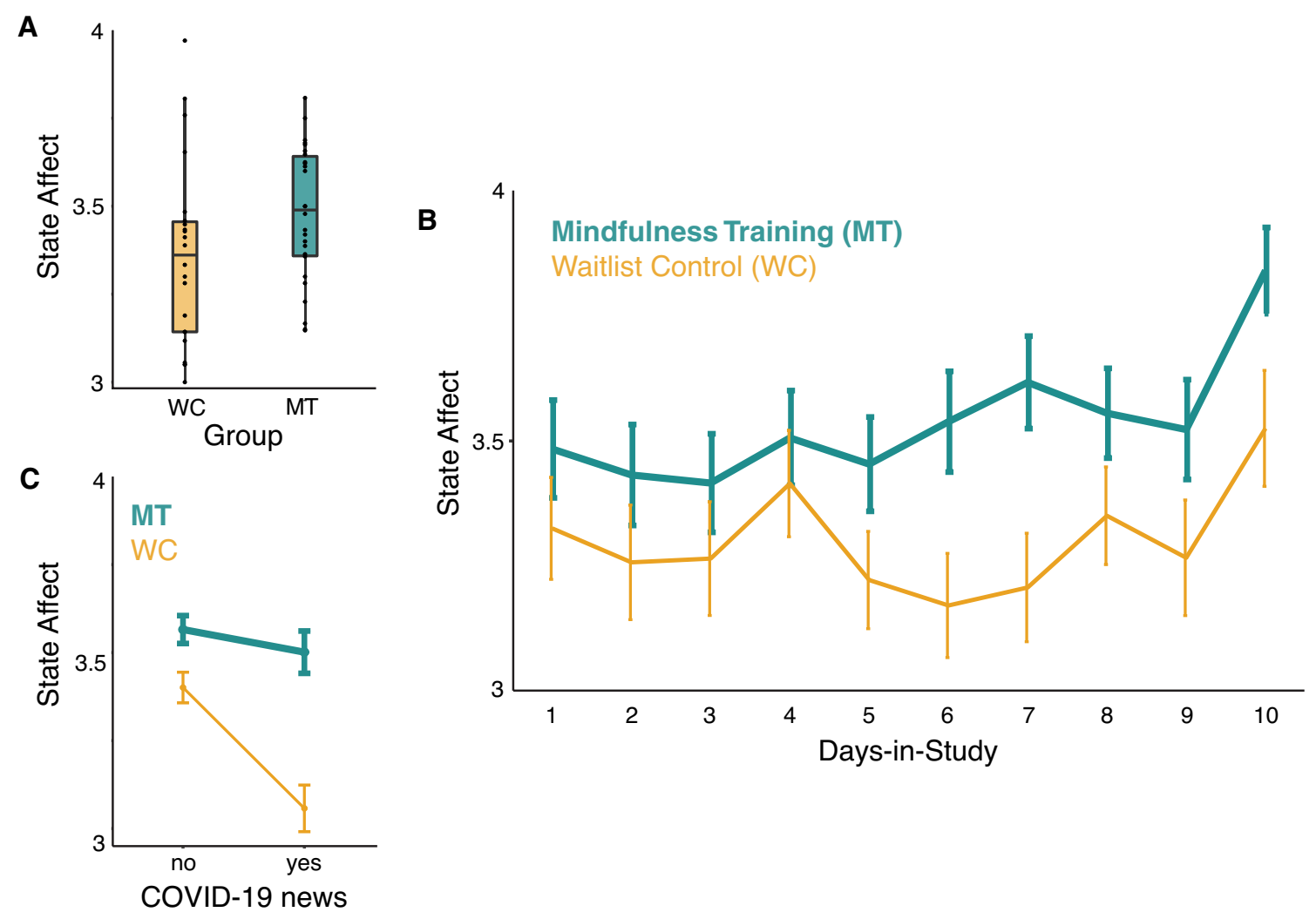

Fig. 1 Group differences in state affect assessed via ecological momentary assessment (EMA) as a function of day and exposure to COVID-19-related news. Response scale of state affect in EMA ranges from 1 (extremely negative) to 5 (extremely positive). A Mindfulness training (MT) group reported more positive affective

positive affective valence in the MT group compared to the WC group ( $p=0.004$, Cohen's $\mathrm{d}=0.28$; as shown in Fig. 1A). There was also a main effect of day-in-study, with state affect improving over time ( $p=0.025$, as shown in Fig. 1B). As state affect appears to show a steeper increase in the MT group relative to the WC group, we ran a separate analysis to examine group differences in affective changes over time in the study. The group $x$ day-in-study interaction term was not significant $(b=-0.02, S E=0.02$, $\left.95 \% C I=[-0.01,0.05], p=0.207 ; \chi^{2}(1)=1.60, p=0.206\right)$.

Given the negative impact of news exposure on affect (de Hoog \& Verboon, 2020; Unz et al., 2008), we also examined whether mindfulness training influenced the relationship between news and state affect. Analyses revealed a main effect of news exposure on state affect $(p<0.001)$, wherein exposure to COVID-19-related news within the two hours prior to each probe was associated with more negative affective valence. There was also a significant interaction between group and news exposure on state affect ( $p=0.036$, as shown in Fig. 1C). Follow-up analyses within each group revealed that exposure to COVID-19-related news was associated with more negative affective valence in the WC valence (averaged across time) compared to waitlist control (WC) group. B Changes in the valence of state affect over time (i.e. dayin-study) plotted separately for the MT and WC groups. C The WC group showed more negative affective valence following exposure to COVID-19-related news; however, the MT group did not

group ( $p<0.001$, Cohen's $d=0.41$ ). In contrast, there was no significant relationship between news and state affect in the MT group ( $p=0.223$ ). Removal of age and gender in these models did not change the direction or significance of the effects. Table 3 reports statistical values associated with these linear mixed effects regression models.

We implemented two control analyses to address potential explanations for this group effect. First, this group difference in state affect could possibly be explained by participants simply not paying attention to their task-at-hand, which has been associated with negative affect (Killingsworth \& Gilbert, 2010). To ensure the relationship between news exposure and state affect cannot be accounted for by merely being inattentive, we controlled for task-related attention (i.e. attention is focused on task-at-hand versus on taskunrelated thoughts) by including this measure in the model. This did not change the pattern of results-news exposure still significantly predicted more negative affective valence in the WC group, but not the MT group. Second, it is possible this group difference in state affect associated with COVID-19-related news exposure existed prior to the study and therefore cannot be attributed to training. To rule out 
Table 1 Demographic information for the mindfulness training and waitlist control group

\begin{tabular}{|c|c|c|}
\hline & $\begin{array}{l}\text { Mindfulness training } \\
\text { group }(n=32)\end{array}$ & $\begin{array}{l}\text { Waitlist } \\
\text { control group } \\
(n=30)\end{array}$ \\
\hline Age $[n(\mathrm{SE})]$ & $30.91(0.37)$ & $28.73(0.10)$ \\
\hline Gender $[\mathrm{f} / \mathrm{m}]$ & $27 / 5$ & $25 / 5$ \\
\hline \multicolumn{3}{|l|}{ Ethnicity $[n(\%)]$} \\
\hline African American & $0(0)$ & $0(0)$ \\
\hline Asian & $5(15.6)$ & $8(26.7)$ \\
\hline White & $20(62.5)$ & $19(63.3)$ \\
\hline Latino or Hispanic & $2(6.3)$ & $0(0)$ \\
\hline Native American & $0(0)$ & $0(0)$ \\
\hline Pacific Islander & $0(0)$ & $0(0)$ \\
\hline Two or more above & $2(6.3)$ & $1(3.3)$ \\
\hline Other & $3(9.4)$ & $2(6.7)$ \\
\hline \multicolumn{3}{|l|}{ Education $[n(\%)]$} \\
\hline High School & $3(9.4)$ & $0(0)$ \\
\hline Bachelor & $12(37.5)$ & $14(46.7)$ \\
\hline Masters & $14(43.8)$ & $15(50)$ \\
\hline Doctoral Degree & $1(3.1)$ & $1(3.3)$ \\
\hline Medical Degree & $1(3.1)$ & $0(0)$ \\
\hline Trade school & $1(3.1)$ & $0(0)$ \\
\hline
\end{tabular}

The two groups did not significantly differ on any of these demographic variables

this alternative explanation, we implemented post hoc analyses to examine whether the group $x$ news exposure interaction differed between the first and second half of the study. This interaction was only significant in the second half of the study, indicating that the observed group differences in their affective response to COVID-news exposure could not have been attributed to baseline differences; rather, the group differences were only observed in the later part of the study after days of training thereby providing support that this group difference can be attributed to mindfulness training. Details of these analyses are reported in Supplementary Information.

Finally, we examined the relationship between the four training-related variables during mindfulness training reported daily immediately following practice and state affect assessed via EMA within the MT group. Affective levels following daily practice significantly predicted state affect measured during EMA $(p<0.001)$. Those who spent more time practicing each day also reported more positive affect throughout the 10 days $(p=0.039)$. These dosedependent relationships indicate daily experiences of practicing meditation track with affective dynamics within the MT group. Details of these analyses are reported in Supplementary Information.

\section{Discussion}

The current study assessed the beneficial effects of daily practice of brief mindfulness meditation on affective wellbeing, especially following exposure to COVID-19 news. We compared the affective well-being of participants who practiced guided mindfulness meditation with those who did not, both at the end of training via questionnaires, and throughout the course of the training via ecological momentary assessments. At the end of the 10-day training, participants who practiced mindfulness meditation reported higher levels of positive affect compared to those who did not. However, the two groups did not differ in other affective
Table 2 Four linear regression models testing the effect of group on four post-training assessment measures of affective well-being

\begin{tabular}{lrrrlllll}
\hline $\mathrm{DV}^{\mathrm{a}, \mathrm{b}}$ & \multicolumn{1}{c}{$\beta^{\mathrm{a}}$} & \multicolumn{1}{c}{$b^{\mathrm{a}, \mathrm{c}}$} & $\mathrm{SE}^{\mathrm{c}}$ & $95 \% \mathrm{CI}^{\mathrm{c}}$ & $p^{\mathrm{c}}$ & $F(4,57)^{\mathrm{d}}$ & $p^{\mathrm{d}}$ & $\operatorname{Adj} R^{2}$ \\
\hline Positive affect & 0.36 & 3.32 & 1.57 & {$[0.17,6.47]$} & .039 & 21.09 & $<.001$ & 0.57 \\
Negative affect & 0.03 & 0.20 & 1.68 & {$[-3.16,3.57]$} & .905 & 4.11 & .005 & 0.17 \\
Depression & -0.07 & -0.51 & 1.52 & {$[-3.55,2.53]$} & .739 & 6.55 & $<.001$ & 0.27 \\
Anxiety & 0.12 & 0.93 & 1.65 & {$[-2.38,4.25]$} & .575 & 9.66 & $<.001$ & 0.36 \\
\hline
\end{tabular}

Each row presents results from one linear regression model, with each model containing one of the four dependent variables assessed post-training. Each regression model consisted of the independent variable, group, which was coded as $1=$ waitlist controls $(n=30)$ and $2=$ mindfulness training $(n=32)$. All models also included age, gender (coded as $1=$ female and $2=$ male), and the same measure at pre-training baseline assessment as covariates

${ }^{a} \mathrm{DV}=$ dependent variable. $\boldsymbol{\beta}=$ standardized coefficient. $\mathrm{b}=$ unstandardized coefficient. $\mathrm{SE}=$ standard error of the mean

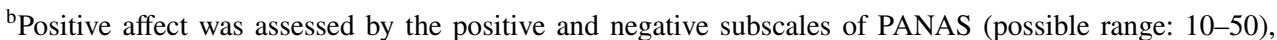
depression and anxiety were assessed by the PROMIS-depression scale and PROMIS-anxiety scale (possible range: $8-40$ )

${ }^{\mathrm{c}}$ Standard errors of the mean, 95\% CI (confidence interval) and $p$ value associated with the unstandardized coefficient

${ }^{\mathrm{d}} p$ value associated with the $\mathrm{F}$ statistic 
Table 3 Linear mixed effects regression models predicting state affect measured in ecological momentary assessment data

\begin{tabular}{lrrllrl}
\hline $\mathrm{IV}^{\mathrm{a}}$ & \multicolumn{1}{c}{$\beta^{\mathrm{a}}$} & \multicolumn{1}{c}{$b^{\mathrm{a}, \mathrm{b}}$} & $\mathrm{SE}^{\mathrm{a}, \mathrm{b}}$ & $95 \% \mathrm{CI}^{\mathrm{b}}$ & $\chi^{2}(1)^{\mathrm{c}}$ & $p^{\mathrm{c}}$ \\
\hline Group & 0.39 & 0.36 & 0.10 & {$[0.16,0.55]$} & 8.47 & 0.004 \\
No news & 0.33 & 0.30 & 0.08 & {$[0.15,0.45]$} & 12.97 & $<0.001$ \\
Day-in-study & 0.05 & 0.02 & 0.01 & {$[0.002,0.03]$} & 4.99 & 0.025 \\
Group x no news & -0.24 & -0.21 & 0.10 & {$[-0.41,-0.01]$} & 4.41 & 0.036 \\
No news in WC & 0.34 & 0.30 & 0.08 & {$[0.15,0.45]$} & 15.76 & $<.001$ \\
No news in MT & 0.09 & 0.08 & 0.07 & {$[-0.05,0.21]$} & 1.47 & 0.225 \\
\hline
\end{tabular}

Note: The first four rows present four independent variables' effects on the dependent variable of state affect (ranging from $1=$ extremely negative to $5=$ extremely positive) from a single linear mixed effects regression model ( $n=1638$ observations). Independent variables included group $(1=$ waitlist controls, $n=26$ and $2=$ mindfulness training, $n=32$ ), news $(1=$ COVID-19 news exposure, $2=$ no COVID-19 news exposure), day-in-study (range from 1 to 10 for each day of study), and group by news interaction. The model also included age and gender (coded as $1=$ female and $2=$ male) as covariates. The last two rows present results from two linear mixed effects regression models that follows up on the group $\mathrm{x}$ news interaction effect, separately implemented within the $\mathrm{WC}=$ waitlist control group $(n=731$ observations) and MT $=$ mindfulness training group ( $n=907$ observations)

${ }^{a} \mathrm{IV}=$ independent variables. $\boldsymbol{\beta}=$ standardized coefficient. $\mathrm{b}=$ unstandardized coefficient. $\mathrm{SE}=$ standard error of the mean

${ }^{b}$ Standard errors of the mean and $95 \%$ CI (confidence interval) associated with the unstandardized coefficient

${ }^{c} p$ value associated with the $\chi^{2}$-statistic, which tests the current model against a null model without the independent variable of interest measures, including negative affect, anxiety and depression. Further, within the mindfulness training group, individuals who practiced more demonstrated the greatest increase in positive affect in EMA surveys. Throughout the study, the mindfulness training group also reported more positive affect on a daily basis compared to the waitlist control group. Importantly, participants' state affect was not impacted by exposure to COVID-19-related news. In contrast, those who did not practice mindfulness meditation reported more negative affect following COVID-19 news exposure. Taken together, brief daily practices of mindfulness meditation led to a modest increase in positive affect, and acted as a protective factor against the negative impact of exposure to COVID-19-related news on affective well-being.

In our study, brief daily practice of mindfulness meditation led to an increase in positive affect. This finding is consistent with previous reports of improved affective wellbeing following mindfulness training (Khoury et al., 2015; Schumer et al., 2018). In particular, studies that involved shorter term training (Schumer et al., 2018) as in our study reported smaller effect sizes in affective well-being, in line with our observation, as compared to studies involving longer periods of training that reported larger effect sizes (Goyal et al., 2014; McClintock et al., 2019). One novel aspect of our study pertains to our experimental approach involving random assignment that was situated in participants' everyday life in which they have agency over their preferred 10-h time window for receiving EMA surveys, as well as the timing, duration, and course option for the mindfulness intervention, highlighting the ecological validity of these findings. Notably, while ample studies support the positive impact of mindfulness training, some caution that mindfulness-based intervention as with any other intervention is not universally beneficial (Britton, 2019; Grant \& Schwartz, 2011). Instead, its effects show a non-monotonic, inverted U-shaped trajectory, suggesting factors such as individual differences (Rojiani et al., 2017), social contexts (Lindahl et al., 2019), or an individual and context interaction modulate the direction and magnitude of these benefits. On one end, those who have a current diagnosis of any psychiatric disorders are discouraged from participating in mindfulness-based interventions because such interventions, if not designed to address specific clinical issues, can lead to deterioration of the disorders (Santorelli, 2014). In contrast, individuals with little or no depressive symptoms may show less improvement due to floor effects (Roos et al., 2017). Most of the participants in our study reported average or above-average levels of affective well-being, with only a small percentage reporting clinical levels of depression and anxiety at baseline. Taken together, the positive impact of brief mindfulness meditation on affective well-being may be modest; our study nonetheless indicates that even short bouts of daily mindfulness practice have the capacity to enhance our affective experience amidst the COVID-19 pandemic.

Our results indicated that daily practice of mindfulness meditation protected individuals against the impact of COVID-19-related news exposure on state affect. Participants who did not undergo mindfulness meditation reported more negative affect following exposure to COVID-19-related news. These results are in line with a recent finding 
that indicates the consumption of COVID-19 news is associated with the largest reduction in positive affect compared to other activities measured (Lades et al., 2020). However, those who underwent mindfulness meditation in our study did not show this negative impact. We ruled out potential confounding factors that could account for this relationship, such as group differences in frequency of news exposure, and inattention to the ongoing task at hand (which at times has been associated with negative affect; Killingsworth \& Gilbert, 2010). One potential mechanism by which mindfulness training protects one against the harmful effects of news exposure include an improved capacity to regulate physiological response to stressors (Shearer et al., 2015) and reduced emotional reactivity (Gu et al., 2015; Wenzel et al., 2020). Another potential mechanism is via enhanced recovery from negative experiences (Cho et al., 2017; Lindsay \& Creswell, 2017), in which mindfulness training helps one accept and let go of negative emotional response to news. Future studies that combine physiological and subjective measures can examine the mechanisms underlying the protective role of mindfulness meditation against the negative affect caused by COVID-19 news exposure.

Several limitations should be considered. First, our study has a relatively small, convenience sample, composed of more females than males. To ensure that participants shared a similar experience of the impact of COVID-19 on their community, we restricted the time window of data collection as new COVID-19 cases began to plateau in the primary site of recruitment. Given this relatively small sample size, our study may have power to detect group differences in post-training measures (as supported by our post hoc power analysis), as well as variables with two levels (e.g., group and news) based on ecological momentary assessments. However, it may be insufficiently powered to detect two-way interactions involving variables that contain multiple levels (e.g., the "day-in-study" variable consists of 10 levels for each day of the training period). Although past studies with similar sample sizes have reported positive findings (Basso et al., 2019; Economides et al., 2018; Shearer et al., 2015), these results need to be replicated with a larger sample size as in other studies using ecological momentary assessments (Lindsay et al., 2018; Rowland et al., 2018). Second, our waitlist control group did not have a comparable daily exercise, rendering it possible that our observed effects can be attributed to demand characteristics and expectation effects (Tang et al., 2015). However, these alternative explanations could not fully account for our findings of a dose-dependent relationship in the mindfulness training group, where those who were more engaged during the training showed larger improvements. Third, our study only measured affective valence. In line with the proposition that mindfulness training helps cultivate a dispositional tendency of calmness important for wellbeing (Desbordes et al., 2015), past work has shown that more time spent on practicing mindfulness preferentially enhances low but not high arousal positive emotions, such as quiet and calm (Jones et al., 2018). These findings highlight the value of examining arousal and valence in future studies of mindfulness intervention.

Moreover, although we asked participants to respond within 15 min of receiving the survey, our analysis did include reports completed within $30 \mathrm{~min}$ from the time the survey was sent to ensure sufficient data points. Surveys completed beyond 15 min may not have as accurately reflected the momentary experience. Finally, the duration of mindfulness training in our study is relatively short. Traditional mindfulness training programs that span a longer time interval with in-person visits have reported larger effects on affective well-being (Goyal et al., 2014; McClintock et al., 2019). Nonetheless, meta-analyses of short-term (Schumer et al., 2018) and technology-based (Victorson et al., 2020) mindfulness training have also reported a small but significant impact on improved affect. Accordingly, it is important to acknowledge the benefit of mindfulness training can be observed after and throughout the 10 days of training in our study. Although we cannot determine how long-lasting these benefits are in the absence of follow-up assessments in our study, what our results do indicate is that the brief sessions of mindfulness training correspond with more positive affect in the moment.

In summary, the current study revealed that brief daily practice of guided mindfulness training leads to a modest increase in positive affect, and protects our affective wellbeing from the negative impact of COVID-19 news exposure. It also underscores the unique value of using ecological momentary assessments in examining affect dynamics in the real world. Our findings indicate that there are beneficial effects of brief periods of mindfulness training on momentary affective well-being, highlighting guided mindfulness meditation as a simple-to-implement, cost-effective technique that can be practiced anywhere, anytime.

Supplementary Information The online version contains supplementary material available at https://doi.org/10.1007/s00426-021-01550-1.

Acknowledgements We would like to thank our participants for their time and dedication to this study. We also appreciate the reviewers' detailed and thoughtful feedback on our manuscript. JWYK and JJ are supported by the Natural Sciences and Engineering Research Council. LTM is supported by an early career development award from the Canadian Child Health Clinician Scientist Program and an Early Career Investigator Award from the Canadian Institute of Health Research.

Availability of data and code The dataset analysed in the current study as well as analysis code are available from the corresponding author on reasonable request. 


\section{Declarations}

Conflict of interest The authors declare they have no conflicts of interest.

Consent to participate/publish All participants provided informed consent, which involves the consent to publish their data.

Ethics approval The study was approved by the Conjoint Faculties Research Ethics Board at the University of Calgary and was performed in accordance with the ethical standards as laid down in the 1964 Declaration of Helsinki and its later amendments or comparable ethical standards.

\section{References}

Basso, J. C., McHale, A., Ende, V., Oberlin, D. J., \& Suzuki, W. A. (2019). Brief, daily meditation enhances attention, memory, mood, and emotional regulation in non-experienced meditators. Behavioural Brain Research, 356, 208-220. https://doi.org/10. 1016/j.bbr.2018.08.023

Bishop, S., Lau, M., Shapiro, S., Carlson, L., Anderson, N., Carmody, J., Segal, Z., Abbey, S., Speca, M., Velting, D., \& Devins, G. (2004). Mindfulness: A proposed operational definition. Clinical Psycholology Science and Practice, 11, 230-241.

Bostock, S., Crosswell, A. D., Prather, A. A., \& Steptoe, A. (2019). Mindfulness on-the-go: Effects of a mindfulness meditation app on work stress and well-being. Journal of Occupational Health Psychology, 24(1), 127-138. https://doi.org/10.1037/ocp0000118

Britton, W. (2019). Can mindfulness be too much of a good thing? The value of a middle way. Current Opinion in Psychology, 28, 159-165. https://doi.org/10.1016/j.copsyc.2018.12.011

Brown, K. W., \& Ryan, R. M. (2003). The benefits of being present: Mindfulness and its role in psychological well-being. Journal of Personality and Social Psychology, 84(4), 822-848. https://doi. org/10.1037/0022-3514.84.4.822

Cella, D., Riley, W., Stone, A., Rothrock, N., Reeve, B., Yount, S., Amtmann, D., Bode, R., Buysse, D., Choi, S., Cook, K., Devellis, R., Dewalt, D., Fries, J. F., Gershon, R., Hahn, E. A., Lai, J. S., Pilkonis, P., \& Revicki, D. (2010). The patient-reported outcomes measurement information system (PROMIS) developed and tested its first wave of adult self-reported health outcome item banks: 2005-2008. Journal of Clinical Epidemiology, 63(11), 1179_ 1194. https://doi.org/10.1016/j.jclinepi.2010.04.011

Cheung, R. Y. M., \& Ng, M. C. Y. (2019). Mindfulness and symptoms of depression and anxiety: The underlying roles of awareness, acceptance, impulse control, and emotion regulation. Mindfulness, 10(6), 1124-1135. https://doi.org/10.1007/s12671-018-1069-y

Chin, B., Lindsay, E. K., Greco, C. M., Brown, K. W., Smyth, J. M., Wright, A. G. C., \& Creswell, J. D. (2019). Psychological mechanisms driving stress resilience in mindfulness training: A randomized controlled trial. Health Psychology, 38(8), 759-768. https://doi.org/10.1037/hea0000763

Cho, S., Lee, H., Oh, K., \& Soto, J. (2017). Mindful attention predicts greater recovery from negative emotions, but not reduced reactivity. Cognition and Emotion, 31(6), 1252-1259.

de Hoog, N., \& Verboon, P. (2020). Is the news making us unhappy? The influence of daily news exposure on emotional states. British Journal of Psychology, 111(2), 157-173. https://doi.org/10. 1111/bjop.12389

Desbordes, G., Gard, T., Hoge, E., Hölzel, B., Kerr, C., Lazar, S., Olendzki, A., \& Vago, D. (2015). Moving beyond mindfulness:
Defining equanimity as an outcome measure in meditation and contemplative research. Mindfulness, 6(2), 356-372.

Eberth, J., \& Sedlmeier, P. (2012). The effects of mindfulness meditation: A meta-analysis. Mindfulness, 3(3), 174-189. https://doi.org/ 10.1007/s12671-012-0101-x

Economides, M., Martman, J., Bell, M. J., \& Sanderson, B. (2018). Improvements in stress, affect, and irritability following brief use of a mindfulness-based smartphone app: A randomized controlled trial. Mindfulness, 9(5), 1584-1593. https://doi.org/10.1007/ s12671-018-0905-4

Flett, J. A. M., Hayne, H., Riordan, B. C., Thompson, L. M., \& Conner, T. S. (2019). Mobile mindfulness meditation: A randomised controlled trial of the effect of two popular apps on mental health. Mindfulness, 10(5), 863-876. https://doi.org/10.1007/ s12671-018-1050-9

Fox, J., Weisberg, S., Price, B., Adler, D., Bates, D., Baud-Bovy, G., Bolker, B., \& et al. (2020). Package 'Car'.

Garland, E. L., Geschwind, N., Peeters, F., \& Wichers, M. (2015). Mindfulness training promotes upward spirals of positive affect and cognition: Multilevel and autoregressive latent trajectory modeling analyses. Frontiers in Psychology, 6, 1-13. https://doi. org/10.3389/fpsyg.2015.00015

Geschwind, N., Peeters, F., Drukker, M., Van Os, J., \& Wichers, M. (2011). Mindfulness training increases momentary positive emotions and reward experience in adults vulnerable to depression: A randomized controlled trial. Journal of Consulting and Clinical Psychology, 79(5), 618-628. https://doi.org/10.1037/a0024595

González-sanguino, C., Ausín, B., Ángel, M., \& Saiz, J. (2020). Mental health consequences during the initial stage of the 2020 Coronavirus pandemic (COVID-19) in Spain. Brain Behavior and Immunity, 87, 172-176.

Goyal, M., Singh, S., Sibinga, E. M. S., Gould, N. F., Rowland-Seymour, A., Sharma, R., Berger, Z., Sleicher, D., Maron, D. D., Shihab, H. M., Ranasinghe, P. D., Linn, S., Saha, S., Bass, E. B., \& Haythornthwaite, J. A. (2014). Meditation programs for psychological stress and well-being: A systematic review and metaanalysis. JAMA Internal Medicine, 174(3), 357-368. https://doi. org/10.1001/jamainternmed.2013.13018

Grant, A., \& Schwartz, B. (2011). Too much of a good thing: The challenge and opportunity of the inverted U. Perspective Psychological Science, 6, 61-76.

Grossman, P., Niemann, L., Schmidt, S., \& Walach, H. (2004). Mindfulness-based stress reduction and health benefits: A meta-analysis. Journal of Psychosomatic Research, 57(1), 35-43. https:// doi.org/10.1016/S0022-3999(03)00573-7

Gu, J., Strauss, C., Bond, R., \& Cavanagh, K. (2015). How do mindfulness-based cognitive therapy and mindfulness-based stress reduction improve mental health and wellbeing? A systematic review and meta-analysis of mediation studies. Clinical Psychology Review, 37, 1-12. https://doi.org/10.1016/j.cpr.2015.01.006

Hofmann, S., Sawyer, A., Witt, A., \& Oh, D. (2010). The effect of mindfulness-based therapy on anxiety and depression: A metaanalytic review. Journal of Consulting and Clinical Psychology, $78,169-183$.

Holman, E. A., Garfin, D. R., \& Silver, R. C. (2014). Boston Marathon bombings, media, and acute stress. Proceedings of the National Academy of Sciences, 111, 93-98.

Holmes, E., O’Connor, R., Perry, V., Tracey, I., Wessely, S., Arseneault, L., \&, , et al. (2020). Multidisciplinary research priorities for the COVID-19 pandemic: A call for action for mental health science. Lancet Psychiatry, 7(6), 547-560.

Jha, A., Krompinger, J., \& Baime, M. (2007). Mindfulness training modifies subsystems of attention. Cognitive Affective and Behavioral Neuroscience, 7(2), 109-119.

Jones, D., Graham-Engeland, J., Smyth, J., \& Lehman, B. (2018). Clarifying the associations between mindfulness meditation and 
emotion: Daily high- and low-arousal emotions and emotional variability. Applied Psychology: Health and Well-Being, 10(3), 504-523.

Kabat-Zinn, J. (1990). Full catastrophe living: Using the wisdom of your mind to face stress, pain and illness. New York: Delacorte Press.

Khoury, B., Sharma, M., Rush, S. E., \& Fournier, C. (2015). Mindfulness-based stress reduction for healthy individuals: A metaanalysis. Journal of Psychosomatic Research, 78(6), 519-528. https://doi.org/10.1016/j.jpsychores.2015.03.009

Killingsworth, M. A., \& Gilbert, D. T. (2010). A wandering mind Is an unhappy mind. Science, 330, 932.

Kuppens, P., Oravecz, Z., \& Tuerlinckx, F. (2010). Feelings change: Accounting for individual differences in the temporal dynamics of affect. Journal of Personality and Social Psychology, 99, 1042-1060.

Lades, L., Laffan, K., Daly, M., \& Delaney, L. (2020). Daily emotional well-being during the COVID-19 pandemic. British Journal of Health Psychology, 25(4), 902-911.

Lahtinen, O., \& Salmivalli, C. (2020). The relationship between mindfulness meditation and well-being during 8 weeks of ecological momentary assessment. Mindfulness, 11(1), 255-263. https://doi. org/10.1007/s12671-019-01248-x

Lai, J., Ma, S., Wang, Y., Cai, Z., Hu, J., Wei, N., Wu, J., Du, H., Chen, T., Li, R., Tan, H., Kang, L., Yao, L., Huang, M., Wang, H., Wang, G., Liu, Z., \& Hu, S. (2020). Factors associated with mental health outcomes among health care workers exposed to coronavirus disease 2019. JAMA Network Open, 3(3), e203976. https://doi.org/ 10.1001/jamanetworkopen.2020.3976

Lapate, R. C., \& Heller, A. S. (2020). Context matters for affective chronometry. Nature Human Behaviour Hum Behavior, 4, $688-689$.

Lindahl, J. R., Britton, W. B., Cooper, D. J., \& Kirmayer, L. J. (2019). Challenging and adverse meditation experiences: toward a personcentered approach. In M. Farias, D. Brazier, \& M. Lalljee (Eds.), The Oxford Handbook of Meditation.

Lindsay, E. K., Chin, B., Greco, C. M., Young, S., Brown, K. W., Wright, A. G. C., Smyth, J. M., Burkett, D., \& Creswell, J. D. (2018). How mindfulness training promotes positive emotions: Dismantling acceptance skills training in two randomized controlled trials. Journal of Personality and Social Psychology, 115(6), 944-973. https://doi.org/10.1037/pspa0000134

Lindsay, E., \& Creswell, J. (2017). Mechanisms of mindfulness training: Monitor and acceptance theory (MAT). Clinical Psychology Review, 51, 48-59.

Luo, M., Guo, L., Yu, M., Jiang, W., \& Wang, H. (2020). The psychological and mental impact of coronavirus disease 2019 ( COVID19) on medical staff and general public-A systematic review and meta- analysis. Psychiatry Research, 291, 113190.

McClintock, A. S., Rodriguez, M. A., \& Zerubavel, N. (2019). The effects of mindfulness retreats on the psychological health of nonclinical adults: A meta-analysis. Mindfulness, 10(8), 1443-1454. https://doi.org/10.1007/s12671-019-01123-9

Mills, C., Raffaeli, Q., Irving, Z., Stan, D., \& Christoff, K. (2018). Is an off-task mind a freely-moving mind? Examining the relationship between different dimensions of thought. Consciousness and Cognition, 58, 20-33.

Pappa, S., Ntella, V., Giannakas, T., Giannakoulis, V. G., Papoutsi, E., \& Katsaounou, P. (2020). Prevalence of depression, anxiety, and insomnia among healthcare workers during the COVID-19 pandemic: A systematic review and meta-analysis. Brain Behavior and Immunity, 88(May), 901-907. https://doi.org/10.1016/j.bbi. 2020.05.026

Pilkonis, P. A., Choi, S. W., Reise, S. P., Stover, A. M., Riley, W. T., \& Cella, D. (2011). Item banks for measuring emotional distress from the patient-reported outcomes measurement information system (PROMIS): Depression, anxiety, and anger. Assessment, 18, 263-283.

Pilkonis, P. A., Yu, L., Dodds, N. E., Johnston, K. L., Maihoefer, C. C., \& Lawrence, S. M. (2014). Validation of the depression item bank from the patient-reported outcomes measurement information system (PROMIS) in a three-month observational study. Journal of Psychiatric Research, 56, 112-119.

Rojiani, R., Santoyo, J. F., Rahrig, H., Roth, H. D., \& Britton, W. B. (2017). Women benefit more than men in response to collegebased meditation training. Frontiers in Psychology, 8, 1-11. https://doi.org/10.3389/fpsyg.2017.00551

Romero, C. S., Catalá, J., Delgado, C., Ferrer, C., Errando, C., Iftimi, A., Benito, A., De Andres, J., \& Otero, M. (2020). COVID-19 Psychological Impact in 3109 Healthcare workers in Spain: The PSIMCOV Group. Psychological Medicine. https://doi.org/10. 1017/S0033291720001671

Roos, C., Bowen, S., \& Witkiewitz, K. (2017). Baseline patterns of substance use disorder severity and depression and anxiety symptoms moderate the efficacy of mindfulness-based relapse prevention. Journal of Consulting and Clinical Psychology, 85, 1041-1051.

Rowland, Z., Wenzel, M., \& Kubiak, T. (2018). A mind full of happiness: How mindfulness shapes affect dynamics in daily life. Emotion, 20(3), 436-451. https://doi.org/10.1037/emo0000562

Santorelli, S. (2014). Mindfulness-based stress reduction (MBSR): Standards of practice. Center for Mindfulness in Medicine, Health Care, and Society, University of Massachusetts Medical School.

Schumer, M., Lindsay, E., \& Creswell, J. D. (2018). Brief mindfulness training for negative affectivity: A systematic review and meta-analysis. Journal of Consulting and Clinical Psychology, 86(7), 569-583.

Shearer, A., Hunt, M., Chowdhury, M., \& Nicol, L. (2015). International journal of stress management effects of a brief mindfulness meditation intervention on student stress and heart rate variability effects of a brief mindfulness meditation intervention on student stress and heart rate variability. International Journal of Stress Management, 23(2), 232-254. https://doi.org/10.1037/a0039814

Shiffman, S., Stone, A. A., \& Hufford, M. R. (2008). Ecological momentary assessment. Annual Review of Clinical Psychology, 4, 1-32. https://doi.org/10.1146/annurev.clinpsy.3.022806.091415

Tang, Y., Hölzel, B., \& Posner, M. (2015). The neuroscience of mindfulness meditation. Nature Reviews Neuroscience, 16(5), 312 312. https://doi.org/10.1038/nrn3954

Tang, Y. Y., Ma, Y., Wang, J., Fan, Y., Feng, S., Lu, Q., Yu, Q., Sui, D., Rothbart, M. K., Fan, M., \& Posner, M. I. (2007). Short-term meditation training improves attention and self-regulation. Proceedings of the National Academy of Sciences of the United States of America, 104(43), 17152-17156. https://doi.org/10.1073/pnas. 0707678104

Torales, J., O’Higgins, M., Castaldelli-Maia, J. M., \& Ventriglio, A. (2020). The outbreak of COVID-19 coronavirus and its impact on global mental health. International Journal of Social Psychiatry, 66(4), 317-320. https://doi.org/10.1177/0020764020915212

Unz, D., Schwab, F., \& Winterhoff-Spurk, P. (2008). TV news-the daily horror? Emotional effects of violent TV news. Journal of Media Psychology, 20, 141-155.

Victorson, D. E., Sauer, C. M., Wolters, L., Maletich, C., Lukoff, K., $\&$ Sufrin, N. (2020). Meta-analysis of technology-enabled mindfulness-based programs for negative affect and mindful awareness. Mindfulness, 11(8), 1884-1899. https://doi.org/10.1007/ s12671-020-01373-y

Watson, D., Clark, L. A., \& Tellegen, A. (1988). Development and validation of brief measures of positive and negative affect: The PANAS scales. Journal of Personality and Social Psychology, 54, 1063-1070. 
Wenzel, M., Rowland, Z., \& Kubiak, T. (2020). Like clouds in a windy sky: Mindfulness training reduces negative affect reactivity in daily life in a randomized controlled trial. Stress \& Health, 37, 232-242.

Xiang, Y., Yang, Y., Li, W., Zhang, L., Zhang, Q., Cheung, T., \&, , et al. (2020). Timely mental health care for the 2019 novel coronavirus outbreak is urgently needed. Lancet Psychiatry, 7(3), 228-229.
Publisher's Note Springer Nature remains neutral with regard to jurisdictional claims in published maps and institutional affiliations. 